\title{
Article \\ Causal Reasoning and Event Cognition as Evolutionary Determinants of Language Structure
}

\author{
Peter Gärdenfors ${ }^{1,2} \mathbb{D}$ \\ 1 Department of Philosophy and Cognitive Science, LUX, Lund University, Box 192, S-221 00 Lund, Sweden; \\ Peter.Gardenfors@lucs.lu.se \\ 2 Palaeo-Research Institute, University of Johannesburg, P.O. Box 524, Auckland Park ZA-2006, South Africa
}

Citation: Gärdenfors, P. Causal Reasoning and Event Cognition as Evolutionary Determinants of Language Structure. Entropy 2021, 23, 843. https://doi.org/10.3390/ e23070843

Academic Editors: Francis Heylighen, Tomas Veloz, Olaf Witkowski and Daniela Flores

Received: 12 May 2021

Accepted: 21 June 2021

Published: 30 June 2021

Publisher's Note: MDPI stays neutral with regard to jurisdictional claims in published maps and institutional affiliations.

Copyright: (C) 2021 by the author. Licensee MDPI, Basel, Switzerland. This article is an open access article distributed under the terms and conditions of the Creative Commons Attribution (CC BY) license (https:// creativecommons.org/licenses/by/ $4.0 /)$.

\begin{abstract}
The aim of this article is to provide an evolutionarily grounded explanation of central aspects of the structure of language. It begins with an account of the evolution of human causal reasoning. A comparison between humans and non-human primates suggests that human causal cognition is based on reasoning about the underlying forces that are involved in events, while other primates hardly understand external forces. This is illustrated by an analysis of the causal cognition required for early hominin tool use. Second, the thinking concerning forces in causation is used to motivate a model of human event cognition. A mental representation of an event contains two vectors representing a cause as well as a result but also entities such as agents, patients, instruments and locations. The fundamental connection between event representations and language is that declarative sentences express events (or states). The event structure also explains why sentences are constituted of noun phrases and verb phrases. Finally, the components of the event representation show up in language, where causes and effects are expressed by verbs, agents and patients by nouns (modified by adjectives), locations by prepositions, etc. Thus, the evolution of the complexity of mental event representations also provides insight into the evolution of the structure of language.
\end{abstract}

Keywords: causal cognition; event representation; evolution of language; sentence structure; word classes

\section{Introduction}

Human languages show a great deal of variation, but there are features that seem to be universal. In the Chomskyan tradition, such features are postulated to derive from an innate universal grammar. Within this tradition, however, evolutionary accounts of the universal grammar have focused on the role of recursion [1] (or, in Chomsky's later writings, e.g., [2], the rule of 'merge'), but Jackendoff [3] and Heine and Kuteva [4] have presented some more general principles. In this article, my aim is to provide answers to some fundamental questions concerning the structure of language on the basis of an account of the evolution of human causal cognition.

In regards to the structure of language, I focus on three questions:

(1) Why are sentences central units in language?

In all languages, sentences are fundamental units, but evolutionary linguists have hardly considered why this is so. My answer is, in brief, that sentences express events, and events are basic units of human causal thinking.

(2) Why do sentences consist of noun phrases and verb phrases?

My answer to this question also derives from the structure of mental models of events.

(3) Why are words in languages divided into classes?

Again, I propose that common word classes can be explained by how we think about events.

The questions concerning the evolution of the structure of human language become more acute when comparing it with language-trained apes and other animals communicate. For example, bonobos produce sequences of signs without concern for whether they form 
sentences [5]. The complexity of human language is of a different order from what is found in non-human animal communication. The story of how the structure of language emerged in the hominin line is long, and it no doubt involved several steps.

The overarching question is, therefore, what happened to the human mind during our evolutionary past that can explain why our communication exhibits such structural complexity. In this article, my aim is to derive an answer to this question from an account of the evolution of human thinking about causality. My argumentation consists of three main steps: (i) A comparison between humans and non-human primates suggesting that human causal cognition is based on the reasoning about the underlying forces that are involved in phenomena, while other primates hardly understand external forces. I illustrate this by an analysis of the causal cognition required for early hominin tool use (Section 2). (ii) As a further evolutionary step, humans often organise causal relations in terms of events. I put forward a model where a representation of an event contains two entities representing a cause as well as a result, but also entities such as agents, patients, instruments and locations (Section 3). Section 4 is devoted to showing how communication about events yields evolutionary benefits. In particular, it opens up for cooperation about future goals. (iii) The components of the event representation show up in the structure of language, which will make it possible to answer the three questions above. Sentences form natural units since they refer to events (or states). In regards to word classes, causes and effects are expressed by verbs, agents and patients by nouns (modified by adjectives), locations by prepositions, etc. (Section 5). In conclusion, the evolution of the complexity of mental event components also provides clues to the evolution of the complex structure of language (Section 6).

\section{What Is Special about Human Causal Cognition?}

\subsection{Non-Human Primate Reasoning about Causes}

As background to the evolution of causal cognition in hominins, I present a summary of some of the findings concerning the abilities of non-human primates to reason with causes. This area goes back to Köhler [6], who, in his ground breaking experiments on chimpanzee planning, observed that apes had great difficulties in stacking boxes in order to be able to reach a banana that was hanging from the ceiling. He observes that when Sultan, the most cognitively proficient chimpanzee, tried to put a second box on top of a first, instead of doing so "as might seem obvious, began to gesticulate with it, ... he put it beside the first, then in the air diagonally above, and so forth". Similar observations of other apes, led Köhler to the conclusion that "there is practically no statics to be noted in the chimpanzee" ([6] (p. 149) and [7,8]).

When comparing causal cognition in non-human primates and in the hominin clade, it is useful to distinguish between cued (externally signalled) and detached mental representations [6,9]. A cued representation refers to something in the current (or recently experienced) external situation of the experiencer. When, for example, a chimpanzee uses two stones to crack nuts, it represents one of them as support (anvil) and the other as the hammerstone. By contrast, detached representations stand for objects or events that are not present in the subject's current or recent external context and thus cannot trigger the representation directly. (This notion of detachment is related to Hockett's [10] "displacement", which is one of the criteria he uses to characterise what constitutes a language, but his criterion has a behaviourist touch to it).

An example is that when a chimpanzee plans to fish for termites with, it walks away and breaks off a thin stick from a tree, preparing it to fish with [11]. Forming mental images of the manufactured stick and how it is to be used are detached representations that become part of the chimpanzee's plan.

An individual that has detached representations can create an inner world where consequences of different actions or events can be simulated [12-14]. Spreng and Grady ([15], p. 1112) argue that "remembering one's past, imagining one's future, and imagining the thoughts and feelings of others ... are similar in that they all involve simulating an 
experience that is distinct from stimulus-driven behaviour". Such simulations are central to abstract causal reasoning.

Being able to reason from inanimate effects to non-present causes seems, at present, to be unique to humans. There is a plethora of experiments and observations that indicate that primates cannot infer physical causes from their effects (e.g., [16,17]). For example, Cheney and Seyfarth's [16] experiments with vervet monkeys showed that when catching sight of a predator, they emit warning cries. However, the same monkeys do not react to detached visual signs such as the trail of a snake or the carcass of an antelope in a tree, which indicates a leopard in the vicinity. Thus, while non-human primates are dependent on direct physical effects, it seems that the aptitude for causal understanding based on inanimate or indirect sensory cues evolved only in the hominin species [18-20].

The claim that reasoning from effects to non-present causes is unique to humans has been contended by other researchers. For example, in a study by Völter and Call [21] it was shown that chimpanzees, bonobos and gorillas can follow a trail left by a leaking yoghurt cup that was placed out of their sight to locate the yoghurt. A limitation of the result is that the apes did not use the trail when it did not match the food that was displaced-that is, when it could not be linked directly through sight, smell or taste.

Additional support for the thesis that non-human primates cannot reason about causes that are not perceived comes from an experiment by Civelek et al. [22] that examined how children and chimpanzees reasoned about unseen causes. The subjects saw a reward being dropped through an opaque forked tube into one of two cups. A sound signalled in which of the cups the reward was to be found. In one condition, the sound followed the dropping event, indicating that the cue was caused by the reward falling into the cup, and in another condition, the sound preceded the dropping event. Four-year-old children performed better in the first condition than in the second, which suggests that they understood the unseen cause. Chimpanzees and three-year-old children, however, performed at chance in both conditions.

Furthermore, Povinelli [17] presented some experiments which also indicate that chimpanzees (and other primates) have problems reasoning about the effects of gravitation on objects. These experiments have led to further investigations [23-26], and there is an ongoing debate (see [27,28]). Povinelli and Penn ([29], p. 77) conclude that "only humans are capable of second-order relational reasoning, and only humans, therefore, have the cognitive machinery that can support higher-order, theory-like, causal relations". JohnsonFrey ([30], p. 201) also writes: "Comparative studies of chimpanzee tool use indicate that critical differences are likely to be found in mechanisms involved in causal reasoning rather than those implementing sensorimotor transformations".

In a study comparing the nut-cracking performances of humans and chimpanzees [31], the result was that humans understood how to apply force to extract numerous nut species through using hammerstones. In contrast, chimpanzees only applied hammerstones to Panda nuts, although they regularly eat hard Irvingia nuts using their teeth. Chimpanzees in other groups and regions cracked different nut types with hammerstones [32,33], but a single group do not use hammerstones to obtain several food sources. This example illustrates how humans, in contrast to chimpanzees, reason more abstractly about the causal effects of applying tool-assisted forces. This allows humans to generalize a particular solution to a wider range of problems.

Non-human animals can reason about an event involving actions-their own or those of others. The important step in reaching the more general event representation presented in the following section comes when not only actions but also other types of forces can function as causes in events. The ability to reason causally about detached forces-and not just actions, the human mind has evolved an extended capacity to reason and to plan that surpasses that of other primate species. 


\subsection{Human Reasoning about Forces}

The human capacity to reason about of physical forces develops early in infants. Michotte [34] showed that if one object moving on a screen came into contact with another object and the other object began moving in the same direction, then adults perceived a causal relation between the two movements. If the second object only began moving 500 milliseconds after the collision, however, the time difference eliminated the impression of causality. Michotte's experiments were performed with six-month-old infants by Leslie and Keeble [35]. The result was that the infants reacted differently to the two types of events. Leslie [36] argues that infants have a special system in their brains for mapping the "forces" of objects. Wolff and his collaborators [37-41] have collected further evidence supporting that people can directly perceive the forces that lie behind different kinds of events. The upshot is that the sensory input generated by the movements of an object is sufficient for the brain to automatically calculate the forces that lead to the movements [42].

Adults can also combine physical causes in their reasoning, as shown by Wolff [37]. The study showed that they can estimate the combined forces of a boat motor and the wind and to determine how the boat crosses a lake. Göksun et al. [43] extended this to a study of 3-5-year-olds. In addition to one-force events, the children were asked to predict the path of a ball that was influenced by two forces that were combined to reflect force dynamics patterns of "cause", "enable" and "prevent". The result was that while children could successfully reason about the one-force events, they had problems with a second force, incorporating it only if the two forces move in the same direction. The older the children, the more successful they were in accounting for the effects of the second force [44]. These experiments suggest that human abstraction and reasoning about physical forces develop over age, although the general system for perceiving forces as causes is present already at an early age.

As humans, we do not only reason about physical forces but also about how psychological and social factors influence us. The increasing complexity of hominin societies has generated a highly developed "theory of mind", that is, an understanding of how our emotions, desires, intentions and beliefs lead to different kinds of interactions between people [13,45-48]. By observing the actions of ourselves and others and through various processes of social learning, we infer the state of mind of other humans under the hypothesis that their theory of mind is similar to our own.

In such cases, we do not perceive the cause of another's actions physically, but use our understanding of their inner state as a causal variable for their behaviours. This involves a separation of perceptual similarities from the causal ones that are determined from emotions, desires, intentions and beliefs. Thus, the mental entities form a class of hidden variables that act as social forces and which we add to our perception in order to understand causal relations. A theory of mind is, therefore an important extension of human causal cognition [49].

\subsection{Tool Manufacture and Use Were Selective Factors for Reasoning about Forces}

From an evolutionary perspective, the central question becomes: Why did only hominins evolve causal thinking that is based on forces? Gärdenfors and Lombard [50] argue that tool manufacture and use were contributing factors to advanced forms of causal reasoning. The key to the argument is that tools extend the potential of the hominins to act across space and time.

As regards spatial cognition, the visual field of primates is divided into peri-personal and extra-personal space. The peri-personal space (the region within reach around the body), makes it possible for an individual to see its field of action. Tool use extends the peripersonal space [51,52]). Even further extensions of the peri-personal space are achieved when the tool leaves direct control of the body and exerts its force at a distance-entering extra-personal space, which may have stimulated the development of causal reasoning about external forces [50]. For example, throwing an object, as many primates do, may be the first method of exerting a force at a distance (see [53] for a speculative account). 
Chimpanzees, however, do not hunt by throwing. When they hunt bushbabies with sharpened sticks, they thrust with direct force [54]. Apes do not have the accuracy, force and speed that make human throwing so effective and so dangerous [55].

Sometime during hominin evolution, the display function of throwing in apes was replaced by a physical function with the aim to hurt or kill prey or opponents. A thrower that could reason about the physical effects at a distance of a stone or a spear would be more successful in hunting or warfare than an individual that could not [56]. The transition from actions to forces as causes is a critical step in the evolution of causal reasoning that can be traced through the use of hunting tools and weapons. The mapping between cause and effect must be inferred from the consequences of the throw (for example, from the behaviour of an animal or enemy that is hit). From the archaeological record, it would seem that Homo neanderthalensis shared the capacity for such reasoning with early Homo sapiens [57-59]. Homo erectus, on the other hand, may have been limited to thrusting weapons $[60,61]$, wherein causal understanding was identified through the stabber's own bodily action.

An even more complex form of causal force is the use of poisoned arrows that operates for an extended period of time, sometimes across a long distance, and often out of the sight of the hunter (see [50,62]. When preparing and using a poisoned arrow, the hunter must rely on advanced forms of abstract causal reasoning and long-term planning.

To sum up this section, non-human animals understand causation only in terms of agency, while humans can also reason about causes in a detached way via forces that operate across space (action at a distance) and time. Among the forces, one also finds the mental variables that are involved in an extended theory of mind.

\section{Event Cognition}

\subsection{A Cognitive Model of Events}

In this section, I turn to the relation between causal thinking and event cognition. A considerable part of human cognition depends on representations of events [63-67]. We use events in causal reasoning, planning and communication. As I argue below, our episodic memory also depends on event structures [66].

A central feature of events is that they are based on causal relations: An event typically contains information about an agent who is the cause of an action that leads to a result related to a patient. Although event representations generally contain an agent, some do not involve any, for example, events of raining, falling, drowning, dying and growing. A representation of an event may also contain other "thematic roles" such as instrument, recipient and beneficiary [68,69]. Agents and patients are object categories with different properties. It is assumed that an agent is able to act, which in the proposed framework amounts to exerting forces. The core idea of the event model presented in [63] and [65] is that an event contains two vectors-the force of an action that drives the event, and the result of the force (see Figure 1) (More formal details of the model can be found in [63-65]).

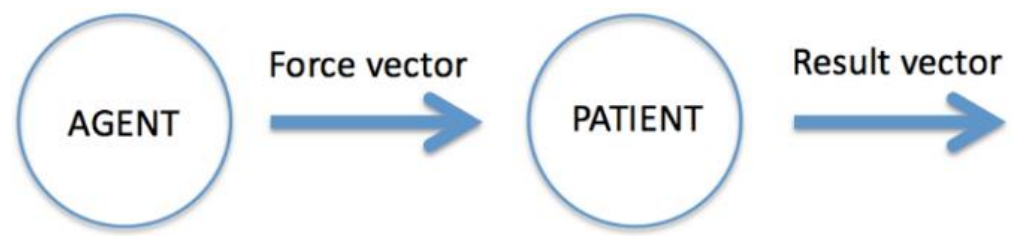

Figure 1. The main components of an event representation.

An action is modelled as a force vector (or a pattern of force vectors as in running). The result of an event is modelled as a change vector representing the change of properties of the patient [63,67]. For example, when Oscar (the agent) pushes (the force vector) a table (the patient), the force exerted makes the table move (the result vector). Or, when Victoria boils the carrots, the result is that the carrots become soft. When the result vector is just a point (a null vector), that is, when the result is no change, then the event is a state. An 
important feature of the event model is that it captures a basic sense of causation: The action of the agent causes the change in the patient. The distinction between forces and changes of states [37,39], results in the fundamental division between causes and effects. A special case of the event model, expressed linguistically by intransitive constructions such as "Victoria is walking" and "Oscar is jumping", is when the patient is identical with the agent. In this case, the agent exerts a force on itself. In other words, the agent modifies its own position or property in some domain of agent space (=patient space).

In philosophy and psychology, the causal relationship between the action and the effect is typically analysed as being between two events (see [70,71]). In contrast, the proposed model describes causation as a relation within an event. Furthermore, in contrast to traditional philosophical theories, the distinction between forces and changes of states also entails that the cause and the result are represented as two different kinds of entities.

To be sure, humans also reason about another form of causation, that is, generic causal relations, for example, "eating toadstools will make you sick" and "lions kill people" [72]. Such generics concern relations between concepts and not causation within single events. There exist many attempts within economics and philosophy to reduce this kind of causal relation to information theory in terms of the probabilities involved (e.g., [73,74]). However, in the case of "lions kill people" the probability of a lion killing someone is much lower than many other causes of death, so the content of the generic is very low if measured in terms of general information or entropy. Gärdenfors and Osta Vélez [72] argue that the strength of the generic depends on the frequency of the characteristic category that is in focus relative to a contrast class. In the case of "lions kill people", this would mean that the proportion of lions that kill people is higher than the corresponding proportion for most other animals. The relevant contrast class is, however, context-dependent so that transforming this into an information-theoretic measure would involve several factors. I will return to the role of generics in Section 5.1.

Another aspect is that the forces are not the only components involved in human causal reasoning, but counterforces (forces exerted by the patient or contextual forces such as gravitation) are also accounted for. This aspect was pioneered in Talmy's [75] "force dynamics" and is further developed in Wolff's [37-39] dynamics model. Depending on how the "affector" force vector (produced by an agent) is related to a "patient" force vector to generate a result vector, subjects judge that the affector force either causes, enables or prevents an effect. These results indicate that subjects make a distinction between different kinds of causal relations. Talmy's force dynamics is grounded in physical events, but it can also be used to represent psychological or social forces where components of a theory of mind are exploited.

\subsection{Event Cognition, Planning and Episodic Memory}

A central question is what have been the evolutionary selective factors that resulted in the extended human dependence on mental representations of events. A main part of the answer is that detached event cognition allows us to speculate about potential outcomes of actions, test and re-adjust our imaginative hypotheses, and shift attention from one target to another. It thereby allows generalisation by comparing the force and result in one event with those of another [64].

In particular, different forms of planning involve event cognition. A plan consists of a series of imagined actions as causes together with the expected effects of the actions. For example, a hunter imagines a series of events, some related to the previous movements of an animal, some as part of a plan to kill or catch it. An anthropological example is that when hunting with poisoned arrows, Kalahari San engage in "speculative tracking", using working hypotheses gained from the signs left by an animal, socially and experientially gained knowledge about the behaviours of the animal and of the landscape in which the tracking is taking place [76]. This imagination may also involve an understanding of the mental state of the animal, for example, that the animal is overheated or dehydrated. Based on these imagined reconstructions, the hunter creates predictions in ever changing 
circumstances involving a continuous cognitive process [77]. The upshot is that event cognition allows for ever more complex causal thinking to evolve.

One must, however, distinguish between immediate planning for present goals and prospective planning for future goals (Gulz [78] calls prospective planning anticipatory planning, a term that was also used in $[13,79])$. The crucial distinction is that for an individual to be capable of prospective planning, it must have a detached representation of its future goals. In contrast, immediate planning only depends on the current goals.

Several researchers have argued that the prospective skill for planning for future needs is exclusive to humans (e.g., [6,80-82]). This has been called the Bischof-Köhler hypothesis. In the light of recent experimental results, the hypothesis can, however, no longer be upheld. Great apes are not only able to select tools for future use [83,84], but also to save tools that have currently been used to obtain future goals [85]. The studies strongly suggest that great apes are able to outcompete current drives in favour of future ones $[84,86]$ (This ability to plan for future needs also seems to have evolved independently in the avian taxon of corvids $[87,88])$.

My hypothesis that humans have more sophisticated cognitive representations of events than other species fits well with the theory that humans have an advanced episodic memory, allowing us to remember single events and the order in which they occurred [89,90].

The main neural correlate for both prospective planning and episodic memory is the hippocampal complex (hippocampus together with entorhinal cortex). Brain imaging shows that this complex is active in humans, both when they recall past events and when they imagine future ones [91]. The hippocampus can map out planned spatial paths, but also paths in other conceptual spaces [92,93]. This connection may be part of the explanation of why the hippocampus is central to both navigation and episodic memorytwo seemingly unrelated cognitive capacities.

Related to episodic memory, the event model can handle what-if questions, that is, counterfactual reasoning concerning what would have happened if an action would have been different [94]. For example: "If I had hit the flint core less hard, it would not have broken". Counterfactual reasoning seems to develop fully only relatively late in childhood. To wit, Rafetseder et al. [95] found that such reasoning is not fully developed in all children before 12 years of age, some of whom still lack an understanding of events that are causally dependent on counterfactual assumptions. According to Markovits' [96] developmental patterns of conditional reasoning, fully developed counterfactual causal understanding is only reached between the ages of 14 and 16 (also see [97] on adolescent brain development in relation to counterfactual reasoning).

Apart from counterfactual reasoning, humans can also reason in terms of omissive causation, which concerns events that do not occur. For example, the fact that a hunter did not bring his spear caused him to be attacked by a lion. For many other models of causation, it is difficult to explain omissive causation, but the event model can also handle this (see $[41,75,98])$.

\section{How Event Cognition Improves Communication}

After these presentations of causal cognition and its role in mental representations of events, it is time to discuss the role of these systems in the evolution of language. Before I turn to how they influence the basic structure of language, this section is devoted to a presentation of what I view as the main evolutionary benefits of event representations in communication.

First of all, there are several levels of communication that are important to distinguish in an evolutionary setting. They will be introduced in Section 4.1. Next, symbolic words allow communication about things that are not present in the context. This will be the topic of Section 4.2. Many types of communicative tasks can be completed by using single words or a combination of a few words (or iconic signs). There are, however, two types of communicative situations, both unique to humans, where more complex linguistics 
structures are employed, namely cooperation for future goals (Section 4.3) and narratives (Section 4.4).

\subsection{Levels of Communication}

It is useful to distinguish three levels of communication, in addition to a basic level of cooperation.

Level 0: Praxis. On this level, individuals interact with each other without using intentional communication.

If everybody in a cooperating group performs their assignments as expected, there is no need for explicit communication.

Level 1: Evaluation and instruction. On this level, valuations of the actions of others and of objects can be expressed by non-verbal approval or disapproval. The coordination of actions is achieved by instruction, expressed by wishes or demands. Linguistically, this is typically achieved by imperatives.

This form of communication is found in non-human animals and is among the first to develop in human infants.

Level 2: Coordination of common ground. On this level, individuals inform each other in order to be better coordinated. This is typically achieved via declaratives, but also questions can be used.

On this level communication in the form of sentences becomes central.

Level 3: Coordination of meanings. On this highest level, individuals negotiate the meanings of words (signs).

This is the most advanced level, which assumes a meta-awareness of how language functions.

In the following, level 2 will be in focus since this is where human communication separates from that of other species.

\subsection{Referring to Absent Objects}

If the goal of collaboration is present in the current environment, for example, food to be gathered or an enemy to be fended off, the individuals need not communicate before acting. In contrast, if the goal is distant in time or space, then a shared representation of the goal must be obtained before cooperation can take place. For example, constructing a common dwelling involves coordinated planning of how to procure the building material and how to collaborate in the construction. Level 2 is therefore essential for cooperation about future goals.

A decisive difference between a symbolic language and the signals employed by animals is that signals only refer to what is present in the environment of the animal. For example, vervets only give warning calls when danger is immediate. Signals are about the surrounding world, while symbols often refer to our inner worlds, that is, to our imaginations, memories, plans and dreams. With the aid of symbols, humans can communicate about things that are not here and now or that may not even exist.

The use of symbols replaces cues from the environment in communication. If somebody has an idea about a common goal, she can use symbolic language to convey her thoughts. Furthermore, symbols make it possible for us to coordinate our knowledge, thereby creating a "common ground" [99] that can be exploited to create new forms of cooperation.

The possibility of referring to detached entities makes new forms of cooperation possible. In this way, a communicative system that makes it possible for members of a group to share mental representations of non-present entities becomes selectively advantageous $[100,101]$.

To some extent, referring to objects that are not present on the scene can be done by just pointing [102]. For example, prelinguistic children about 12 months old can sometimes refer metonymically to an absent person by pointing to a place where that person has recently been or is normally located [103]. Chimpanzees can refer to absent objects by pointing, but only to invisible objects they know to be present [104]. They also point 
imperatively (level 1), while infants point declaratively (level 2) [105]. Pointing gestures are also frequently reintroduced in storytelling. Their function then is to give a visual complement to what the words point to in mental space [106,107] and [108] (p. 40) calls this "deixis at phantasma". In this way, pointing begins to allow a "meeting of minds" [109].

Transiting from an animal signalling system to a fully symbolic language requires several steps. Bickerton [110] and other researchers [111-113], argue that during the evolution of language there was a stage when a protolanguage, which contained only the semantic components of language, was used. These authors, claim that Homo erectus mastered a protolanguage, and it only when Homo sapiens emerged on the scene when a language with a fully grammatical structure evolved. Everett [112] distinguishes between three levels of grammaticality (see [3] for another account of intermediary steps of grammatical evolution). The coordination of the common ground required for the forming of a common plan for future actions can possibly be achieved in a communication system that lacks syntax, that is, in a protolanguage. Nevertheless, as I argue in Section 5, some grammatical structure is needed in order to solve problem related to role assignments.

\subsection{Communication for Future Cooperation}

There exist many forms of cooperation in animals, for example, cooperative hunting, but the planning involved is individual, bound to the current context, and is not communicated intentionally. Of course, animals are reading the behaviour and maybe even the intentions of other individuals and use that to determine their own behaviour. This can be seen as a form of communication that improves cooperation, but it is not intentional.

When the planning involves several individuals, then variables of a theory of mind, such as the desires and intentions of other individuals, will also be part of the plan. For example, planning for collaborative hunting typically involves adjusting the plan to the presumed intentions of the other participants.

In contrast, planning for future collaboration seems to be more or less unique to the hominin line $[100,114]$. It essentially involves coordinating goals, which presumes several forms of coordination of common ground: coordination where something will be done (often outside the present visual field), joint reference to absent objects, coordination of goals and coordination of actions. This coordination is improved if the collaborators structure the plans in terms of future events. Thus, the evolution of event cognition made more advanced forms of collaboration possible.

Such planning requires that joint intentions are formed, which is an advanced form of the theory of mind, presumably unique to humans [13,45,48,115]. A joint plan can be analysed as a combination of forming a joint intention and coordinating actions. In previous work $[13,45,79,101,116,117]$. I have argued that symbolic language makes efficient cooperation about future goals possible. Along the same lines, Tylén et al. [118] (p. 6) write:

Analogous to the way that manual tool use has been shown to enlarge the peripersonal space by extending the bodily action potential of arm and hand in space ..., linguistic symbols liberate human interactions from the temporal and spatial immediacy of face-to-face and bodily coordination and thus radically expand the interaction space.

During the evolution that led to Homo sapiens, our hominin ancestors developed new forms of cooperation that made it possible to organise their societies in new ways. It is generally agreed that hominins evolved in open landscapes in which the individual travelled over long ranges [119]. Cooperative foraging could have been caused by increased seasonality and variability in the environments.

The Oldowan culture - the first along the Homo lineage-was signified by an extension in time and space [120]. There is clear evidence that the transport of artefacts (at least stone tools) was an important trait of the culture [121]. Isaac [120] also speculates that division of labour was present in the Oldowan culture. Such arrangements presume coordination of activities, which is an indication of detached communication. The more complex a culture is, the more effort must be made to preserve the complexity. Donald [122] 
writes: "Memory for a variety of special skills usually involves some division of labour, as well as a collaborative strategy for passing those skill on to every new generation". Therefore, teaching about the environment and different procedures for food procuration became increasingly important [123,124].

Division of labour involves role taking. Spontaneous role taking can occasionally be found in social hunting in some non-human species, for example, chimpanzees [125]. If the role taking is flexible, however, so that an individual, for example, sometimes drives the game, sometimes acts as a lookout or sometimes waits in ambush, then each participant must have a representation of the roles of the other team members $[126,127]$. This also involves joint intentionality about the activities of the team [47]. Furthermore, if the role taking is part of a plan, then the roles must be communicated in some way. As I argue in Section 5.2, communication about role assignments maps onto fundamental syntactic principles.

The analysis presented here dovetails with Smith [128] (p. 241), who argues that linguistic communication has the following benefits for cooperation:

(1) Simplifies otherwise difficult coordination problems, especially those involving many agents and planning for future events;

(2) Reduces the cost of enforcing adherence to collectively beneficial norms;

(3) Enhances the efficiency of signals, including those which provide collective goods;

(4) Facilitates the positive assortment of individuals who adhere to similar norms and conventions.

\subsection{Who Did What to Whom? The Adaptive Benefits of Gossip}

In social species, individuals often must decide whether to cooperate or not. Within game theory, in the investigations of prisoners' dilemmas and similar conflict situations, it is taken for granted that the players know who the potential collaborators are. In practice, however, the most important question is: How do you know whom to cooperate with? Dessalles [111] (p. 360) writes: "Some of our ancestors who belonged to the first species of Homo, say, began to form sizeable coalitions. In such a "political" context, finding good allies becomes essential". Sharing information about the other members of the group is an important special case of coordinating common knowledge.

Reciprocal altruism ("you scratch my back, and I'll scratch yours") is found in several animal species. Such cooperation presumes trust between two individuals. Indirect reciprocity is a more extreme form of altruism: "I help you, and somebody else will help $\mathrm{me}^{\prime \prime}$. This form of cooperation involves a group of individuals. The conditions for indirect reciprocity to evolve as an evolutionarily stable strategy have been investigated $[129,130]$. The key concept in Nowak and Sigmund's [129] evolutionary model is that of the reputation of an individual: An individual $X^{\prime}$ s reputation is built up from how members of the society observe X's behaviour towards third parties and then how this information is spread to other members of the society. The higher the reputation of $X$, the more other individuals are willing to cooperate with $\mathrm{X}$.

The communication required for functioning forms of indirect reciprocity typically concerns whom you can trust. The information is generally conveyed in the absence of the individual who is evaluated. This process makes gossip becomes an important way of achieving consensus about reputation [131,132]. In this way, the information that $X$ is a "selfless" helper can be shared knowledge within the group. T X's reputation can then be evaluated by any individual who needs to decide whether or not to assist $X$ in a troublesome situation. Evidently, reputation cannot be directly observed by others in the same way as such status markers as a raised tail among wolves. In contrast, each individual must keep an account of the reputation of the other individuals with whom she considers interacting. Semmann et al. [133] experimentally demonstrate that building a reputation through cooperation is valuable for future social interactions, not only within but also beyond one's social group. Gossip, therefore, plays a central role in the evolution of language. 
Gossip typically contains expressions of the form " $\mathrm{X}$ did A to $\mathrm{Y}$ ", which require thematic roles such as agent, action and patient to be identified. A communication system that communicates this form of messages must be able to (a) refer to individuals in their absence, for example, by names, (b) express that " $X$ was good to $Y$ " and " $Y$ was bad to $X^{\prime \prime}$ and (c) to keep track of the roles of $X$ and $Y$. In order to evaluate $X$ and $Y$ as potential collaborators, such expressions are clear examples of ascribing roles to persons. They are also difficult to convey without ambiguity within a protolanguage: without markers for roles, since " $\mathrm{X}$ was good to $\mathrm{Y}$ " can then not be distinguished from " $Y$ was good to $X$ ". Such a system must thus contain some forms of syntax, maybe of the first type described by Everett [112].

Providing information about whom to cooperate with is a central function of gossip, but not the only one. Dunbar [131] argues that it functions as a replacement for grooming and thus results in stronger bonding between individuals. Furthermore, other forms of information about individuals not related to their trustworthiness can also be important in the complex net of strategic interactions between the members of a group.

I have now presented two forms of communication for cooperation where sentences are required: coordination of future goals and gossip. Both types typically take the form of narratives. Pragmatically, their function is to coordinate the common knowledge of the interlocutors (level 2). I am not claiming that communication on level 2 can only be achieved by sentences. I have already pointed out that pointing and other forms of gestures can also perform this function (see also [134]), and maybe even drawing could achieve this. Nevertheless, sentential structures strongly amplify the efficiency of communication on level 2.

What is important is that describing planned actions as well as information about relations between different individuals are special cases of describing events. My hypothesis is that the capacity to communicate about events is a crucial distinction between the communication of language-trained apes and that of humans.

\section{From Events to Sentences}

In this section, I show how causal cognition and the structure of event representations provide answers to the three questions concerning the fundamental structure of language that were presented in the introduction.

\subsection{Sentences Are Needed for the Coordination of Common Knowledge}

There is one linguistic unit that is central to both types of communication discussed in the previous section: the sentence. The first question on my list concerns why we organise much of our communication in sentences. The Chomskyan tradition takes it for granted that the goal of speaking is to generate sentences, having a minimal structure of a noun phrase and a verb phrase. Within this tradition, the main problem is to decide whether certain combinations of words are grammatical-the meaning of a sentence is secondary. Furthermore, in cognitive linguistics, sentences are seen as natural units [69,135-138].

In analytic philosophy, sentences are also central units. In the tradition since Frege, the content of a sentence is a proposition. As an answer to my first question, Frege writes that the role of sentences is to express thoughts. This answer is simply not sufficient unless it can be determined how a thought is to be identified (independently of language).

If one takes a cognitive perspective on communication, an explanation of why we express ourselves in sentences is needed. My proposal is that the primary function of sentences is that they refer to events. I next outline how a mental representation of an event can be expressed in sentences describing different aspects of the event that are relevant to the communicative situation. Not only what the interlocutors perceive, but also what they are attending to, their current goals, their future plans and their previous communication will influence how sentences are formed.

It is important that I am not claiming that all human communication takes the form of sentences. First of all, from an evolutionary perspective, the focus should be on utterances 
rather than sentences. Utterances can only be evaluated in relation to a communicative context that contributes to their meaning. In contrast, within philosophy (and to a large extent also linguistics), it is assumed that sentences have a meaning that is independent of the context. Second, there several forms of communication, for example, evaluative and emotional expressions, requests and demands, which do not refer to events and which may not even require symbolic structures.

My focus in this article is, however, the communication required for advanced cooperation, and thus primarily for communication on level 2, that is, coordinating inner worlds. For this task, sentences prove their mettle. In present-day human communication, sentential structures are so entrenched in the linguistic structure that they are also used for communication on level 1, which is evaluative expressions and demands, and on level 3 , which is, coordinating meanings of words and other meta-functions of language.

Events are complex phenomena, and they cannot be exhaustively described. Before a sentence can be generated, it must first be decided which information to include. A speaker has the liberty to express different perspectives on the same event. The choice of perspective determines what is expressed in a sentence. In cognitive linguistics, this choice is called the construal of the sentences. One manner of expressing different perspectives is via the choice of grammatical construction. For example, in "Victoria paints Oscar's face", Victoria is more in focus, while in the passive construction "Oscar's face is painted by Victoria" the focus is on Oscar's face. One must therefore distinguish two different levels of meaning: (i) the representation of an event and (ii) a construal that picks out certain aspects of the event.

How is a construal of an event determined? The attention of the speaker is a selection mechanism for a cognitive event representation, as seen in the example above. There are, however, other aspects of how a construal is formed (see [139] (Ch. 3) for a survey). One is perspective: For example, if you and I are standing on two sides of a house, I can say that you are behind the house if I put myself in the centre (egocentric view), or I can say that you are in front of the house if I put the house and the direction of its main side in focus (allocentric view). By analogy with the visual process, a construal focuses only on certain parts of an event. For example, the sentences "Oscar sprayed paint on the wall" and "Oscar sprayed the wall with paint" describe the same event with the aid of two different construals. The difference between them is that in the first, "paint" is focused on as the patient of the action, while in the second, "the wall" is made the patient [69] (p. 124). The attention process is then applied to select the force or the result vector of the event model, generating a construal that can be used in the language production model. For example, in "Oscar scrubs the floor", the force vector is selected for the construal, while in "Oscar cleans the floor", it is the result that is in focus.

On the basis of the notion of a construal of an event, I can now formulate a fundamental connection between the semantics of sentences (utterances) and events. Jackendoff [140] (p. 327) makes a similar proposal: "[T]he category corresponding to a sentence is an event or a state rather than a truth value". However, his event model is algebraic rather than geometric, as proposed here).

Thesis about sentences: A (declarative) sentence expresses a construal of an event.

In linguistics, a tight mapping between linguistic expressions and construals of events is in general assumed [141]. DeLancey [142] provides good linguistic arguments why events must be distinguished from their construals.

The thesis is constrained to declarative sentences. First, on communication level 1 (evaluation and instruction), evaluative expressions or imperatives are typically used instead. Evaluative expressions need not even involve words but can consist of, for example, an affirming nod. Since an imperative relates to the attitudes of the speaker and is normally directed to the addressee, imperatives typically omit some event elements that occur in sentences ("Salt please"). Second, on level 2 (coordination of common ground), a common form of utterances are generics. They have a different function in communication in that they provide information about the underlying semantic structure. Gärdenfors and Osta 
Vélez [72] distinguish between two kinds: (a) Property generics dealing with characteristic properties of objects ("ducks lay eggs"), and (b) event generics dealing with causal relations within event types ("sharks kill people"). Finally, in a seeming violation of the thesis, there also exist declarative sentences used on communication level 2, such as the current one, where information is given without referring to an event. Such sentences, however, communicate abstract thoughts and thereby constitute advanced uses of language (and meta-language) that have evolved at later stages.

\subsection{Noun Phrases and Verb Phrases}

The second question concerns why sentences are built up from noun phrases and verb phrases. In Heine and Kuteva's [4] "layers" of grammaticalisation, nouns constitute the first layer and verbs the second. Nouns and verbs are also the only word classes that are fairly stable cross-linguistically. Why are these classes so fundamental?

I submit that the answer to this question also derives from event cognition to wit, the minimal construals of events. Agents and patients are linguistically expressed by nouns (including names) or noun phrases and force vectors and results vectors by verbs.

Linguists distinguish between manner verbs and result verbs-where "manner verbs specify as part of their meaning a manner of carrying out an action, while result verbs specify the coming about of a result state" [143] (p. 21) (see also [138] (Ch. 1)). This distinction maps directly to the distinction between force vectors and result vectors in events. For example, "push" refers to the force vector of an event, "move" refers to changes in the spatial domain of the result vector and "heat" refers to changes in the temperature domain (Possible exceptions to this general rule are verbs such as "split" that describe changes in which objects exist and verbs such as "give" that describe intentional actions involving recipients. These are discussed in [63] (Section 10.3.2)).

There are four minimal combinations of elements from an event model that are expressed as sentences: (i) agent + force vector ("Oscars scrubs"), (ii) agent + result vector ("Oscar cleans"), (iii) patient + force vector ("the table is scrubbed"), and (iv) patient + result vector (the table is cleaned). Each of these combinations yields a complete sentence. This leads to the following thesis [63] (Ch. 11).

Thesis about event construals: A construal of an event contains at least one vector (force or result) and at least one object (patient or agent).

This thesis requires that at least an agent or a patient (expressed by a noun phrase) and a force vector or a result vector (expressed by a verb phrase) are parts of what is expressed in a sentence. The model thus explains the basic distinction between nouns phrases and verb phrases and why at least one of each typically occurs in a sentence. The thesis thereby provides motivation for why a construction consisting of a noun phrase and a verb phrase is a cognitive unit of communication.

However, the thesis is also supported by studies of grammaticalisation. Heine and Kuteva [4] (p. 119) write: "All evidence from grammaticalisation leads to the same hypothesis, namely that the earliest structure of human language was lexical in nature, first consisting only of noun-like utterances before verbal utterances appeared, thereby making it possible to form propositional constructions".

In linguistics, events are often modelled using symbolic notation [140,144]. For example, Rappaport Hovav and Levin [144] (p.116) represent the meaning of the verb "break" as follows:

\section{[[X $\left.\mathrm{ACT}_{<\mathrm{MANNER}>}\right]$ CAUSE [Become [Y <BROKEN>]]]}

This can be rendered as " $X$ acts in a manner to cause $Y$ to become broken". In this kind of analysis, however, the linguistic level is still present since the verb "break" reappears as $<$ BROKEN $>$.

The ACT-CAUSE-BECOME model of verb semantics presented by Rappaport Hovav and Levin can, nevertheless, be mapped onto the present proposal. The ACT of the formalism corresponds to the force vector, except that not all force vectors involve action. The BECOME corresponds to the result vector (Goddard and Wierzbicka [145] argue that 
"happen to" fits better than "become"). CAUSE is the mapping from force vector to result vector [64]. As should be clear by now, the model presented here is richer since the force and result vectors are grounded in a theory of causal cognition. This connects the semantics of causation and events directly to bodily and perceptual variables.

\subsection{Other Word Classes}

The third question concerns why the words of a language can be grouped into a small number of classes. I have already motivated why there are nouns and verbs since they express the most central components of an event. The basic constituents of events -agent, patient, action and result_-can, however, be augmented with other thematic roles such as recipient ("Oscar handed Victoria a towel") and instrument ("Oscar painted the house with a roller"). Nouns and verbs can, however, be modified in many different ways. Heine and Kuteva [4] show how nouns and verbs have historically been transformed to other word classes. I will only briefly discuss adjectives and prepositions here. The semantic roles of these and other word classes are treated more extensively in the second part of [63].

An adjective has two major communicative functions. The first is as a specification of a noun (or noun phrase) that contributes to the identification of a referent. For example, if you want somebody to fetch you a cup and there are several cups present in the context, you specify it further by adding an adjective, saying "the blue cup" or "the large cup".

The second function of an adjective is informative. You can say, "The stove is hot", as a warning to somebody. In linguistic terms, the adjective is then a complement to a copula ("is") or an intransitive verb ("the meal tastes wonderful"). The two functions of adjectives may very well be cognitively separated. Therefore, it is not necessary that these functions are expressed by one word class. In line with this, Dixon [146] (p. 30) notes that some languages have two different word classes, one fulfilling the specification function and another fulfilling the informative function. As a matter of fact, some words classified as adjectives in English only have one of the functions: "afraid" and "alive" can only be used informatively, and "absolute" and "main" can only be used as a specification [147].

Most prepositions can be grouped into two classes: locative, indicating where something is, and directional, indicating where something is going [148]. Locative prepositions complement a noun (noun phrase) by specifying the location (a region) in the visuospatial domain: "Put the plate in front of grandfather!" This function is required for instruction and is similar to the specification function of adjectives. Another communicative function is handled by directional prepositions. In a sentence such as "Oscar went to the river", the phrase "to the river" has the same function as a result verb: it specifies the result vector of an event. It should be noted that in both functions, the preposition is combined with a noun (or a noun phrase).

In communicating a plan for a collaboration, it is clear that both the specification function of adjectives and the spatial information contained in prepositional constructions add precision to the message. Even though a plan communicated by only nouns and verbs may succeed, the communication typically gains in content by exploiting further word classes.

\section{Conclusions}

In this article, I have argued that the evolution of causal cognition and event representations provides clues to the evolution of the complex structure of language. A consequence of this approach is that the evolutionary processes that led to language were cognitive and social, not primarily linguistic. Donald [122] (p. 2) writes: "If languages are products of cognitive interactions in groups, this fact alone would demand a culture-first theory of language genesis. ... Archaeological evidence suggests that human ancestors were skilled long before they were articulate. ... Therefore the cognitive apparatus for refining skill must have existed in some form before languages could emerge from group interactions". This position entails that most human cognitive functions had been chiselled out by evolution before language appeared on the hominin scene. Language would not have evolved with- 
out these cognitive capacities, in particular advanced causal cognition, having a rich theory of mind, representing events, having a memory system that includes episodic memory and representing future goals $[13,45,49,100]$.

In summary, my main thesis concerning the origins of linguistic structure is that sentences are natural units in communication because human cooperation has benefitted evolutionarily (and still benefits) from communication about events. I have argued that a declarative sentence refers to an event (or a state as a special case). Non-human animals do not refer to events since neither is their causal cognition rich enough nor do they have the mental capacity to cooperate about future goals. Therefore, there has been no selection for communication with a sentential structure in non-human species.

Funding: This research received no external funding.

Data Availability Statement: Not Applicable.

Conflicts of Interest: The author declares no conflict of interest.

\section{References}

1. Hauser, M.D.; Chomsky, N.; Fitch, W.T. The faculty of language: What is it, who has it, and how did it evolve? Science 2002, 298, 1569-1579. [CrossRef] [PubMed]

2. Chomsky, N. Derivation by Phase; MIT Press: Cambridge, MA, USA, 1999.

3. Jackendoff, R. Possible stages in the evolution of the language capacity. Trends Cogn. Sci. 1999, 3, 272-279. [CrossRef]

4. Heine, B.; Kuteva, T. The Genesis of Grammar: A Reconstruction; Oxford University Press: Oxford, UK, 2007.

5. Savage-Rumbaugh, E.S.; Lewin, R. Kanzi: The Ape at the Brink of the Human Mind; Wiley: New York, NY, USA, 1994.

6. Köhler, W. The Mentality of Apes; Penguin Books: Traverse City, MI, USA, 1917.

7. Cacchione, T.; Call, J.; Zingg, R. Gravity and solidity in four great ape species (Gorilla gorilla, Pongo pygmaeus, Pan troglodytes, Pan paniscus): Vertical and horizontal variations of the table task. J. Comp. Psychol. 2009, 123, 168. [CrossRef] [PubMed]

8. Tomonaga, M.; Imura, T.; Mizuno, Y.; Tanaka, M. Gravity bias in young and adult chimpanzees (Pan troglodytes): Tests with a modified opaque-tubes task. Dev. Sci. 2007, 10, 411-421. [CrossRef] [PubMed]

9. Gärdenfors, P. Cued and detached representations in animal cognition. Behav. Process. 1996, 36, 263-273. [CrossRef]

10. Hockett, C.F. The origin of speech. Sci. Am. 1960, 203, 88-96. [CrossRef]

11. Sanz, C.; Morgan, D.; Gulick, S. New insights into chimpanzees, tools, and termites from the Congo Basin. Am. Nat. 2004, 164, 567-581. [CrossRef] [PubMed]

12. Decety, J.; Grèzes, J. The power of simulation: Imagining one's own and other's behavior. Brain Res. 2006, 1079, 4-14. [CrossRef]

13. Gärdenfors, P. How Homo Became Sapiens: On the Evolution of Thinking; Oxford University Press: Oxford, UK, 2003.

14. Grush, R. The architecture of representation. Philos. Psychol. 1997, 10, 5-23. [CrossRef]

15. Spreng, R.N.; Grady, C.L. Patterns of brain activity supporting autobiographical memory, prospection, and theory of mind, and their relationship to the default mode network. J. Cogn. Neurosci. 2010, 22, 1112-1123. [CrossRef]

16. Cheney, D.; Seyfarth, R. Attending to behaviour versus attending to knowledge: Examining monkeys' attribution of mental states. Anim. Behav. 1990, 40, 742-753. [CrossRef]

17. Povinelli, D.J. Folk Physics for Apes: The Chimpanzee's Theory of how the World Works; Oxford University Press: Oxford, UK, 2000.

18. Calvin, W.H.; Bickerton, D. Reconciling Darwin and Chomsky with the Human Brain; MIT Press: Cambridge, MA, USA, 2000.

19. Shaw-Williams, K. The social trackways theory of the evolution of human cognition. Biol. Theory 2014, 9, 16-26. [CrossRef]

20. Stuart-Fox, M. The origins of causal cognition in early hominins. Biol. Philos. 2014, 30, 247-266. [CrossRef]

21. Völter, C.J.; Call, J. Great apes (Pan paniscus, Pan troglodytes, Gorilla gorilla, Pongo abelii) follow visual trails to locate hidden food. J. Comp. Psychol. 2014, 128, 199-208. [CrossRef] [PubMed]

22. Civelek, Z.; Call, J.; Seed, A.M. Inferring unseen causes: Developmental and evolutionary origins. Front. Psychol. 2020, 11, 872. [CrossRef]

23. Call, J. Trapping the minds of apes: Causal knowledge and inferential reasoning about object-object interactions. In The Mind of the Chimpanzee: Ecological and Experimental Perspectives; Lonsdorf, E.V., Ross, S.R., Matsuzawa, T., Goodall, J., Eds.; University of Chicago Press: Chicago, IL, USA, 2010; pp. 75-86.

24. Hanus, D.; Call, J. Chimpanzees infer the location of a reward on the basis of the effect of its weight. Curr. Biol. 2008, 18, R370-R372. [CrossRef]

25. Martin-Ordas, G.; Call, J.; Colmenares, F. Tubes, tables and traps: Great apes solve two functionally equivalent trap tasks but show no evidence of transfer across tasks. Anim. Cogn. 2008, 11, 423-430. [CrossRef]

26. Penn, D.C.; Povinelli, D.J. On the lack of evidence that non-human animals possess anything remotely resembling a 'theory of mind'. Philos. Trans. R. Soc. B 2007, 362, 731-744. [CrossRef]

27. Seed, A.M.; Call, J.; Emery, N.J.; Clayton, N.S. Chimpanzees solve the trap problem when the confound of tool-use is removed. J. Exp. Psychol. Anim. Behav. Process. 2009, 35, 23. [CrossRef] [PubMed] 
28. Seed, A.M.; Hanus, D.; Call, J. Causal knowledge in corvids, primates and children: More than meets the eye? In Tool Use and Causal Cognition; McCormack, T., Hoerl, C., Butterfill, S., Eds.; Oxford University Press: Oxford, UK, 2011 ; pp. 89-110.

29. Povinelli, D.; Penn, D. Through a floppy tool darkly. In Tool Use and Causal Cognition; McCormack, T., Hoerl, C., Butterfill, S., Eds.; Oxford University Press: Oxford, UK, 2011; pp. 69-97.

30. Johnson-Frey, S.H. What's so special about human tool use? Neuron 2003, 39, 201-204. [CrossRef]

31. Boesch, C.; Bombjaková, D.; Boyette, A.; Meier, A. Technical intelligence and culture: Nut cracking in humans and chimpanzees. Am. J. Phys. Anthropol. 2017, 163, 339-355. [CrossRef]

32. Hannah, A.C.; McGrew, W.C. Chimpanzees using stones to crack open oil palm nuts in Liberia. Primates 1987, $28,31-46$. [CrossRef]

33. Morgan, B.J.; Abwe, E.E. Chimpanzees use stone hammers in Cameroon. Curr. Biol. 2006, 16, R632-R633. [CrossRef]

34. Michotte, A. The Perception of Causality; Methuen: New York, NY, USA, 1963.

35. Leslie, A.M.; Keeble, S. Do six-month-old infants perceive causality? Cognition 1987, 25, 265-288. [CrossRef]

36. Leslie, A.M. A theory of agency. In Causal Cognition: A Multidisciplinary Debate; Sperber, D., Premack, D., James Premack, A., Eds.; Oxford University Press: New York, NY, USA, 1995; pp. 121-141.

37. Wolff, P. Representing causation. J. Exp. Psychol. Gen. 2007, 136, 82-111. [CrossRef]

38. Wolff, P. Dynamics and the perception of causal events. In Understanding Events: How Humans See, Represent, and Act on Events; Thomas, S., Zacks, J., Eds.; Oxford University Press: Oxford, UK, 2008; pp. 555-587.

39. Wolff, P. Representing verbs with force vectors. Theor. Linguist. 2012, 38, 237-248. [CrossRef]

40. Wolff, P.; Shepard, J. Causation, touch, and the perception of force. In The Psychology of Learning and Motivation; Ross, B.H., Ed.; Academic Press: New York, NY, USA, 2013; Volume 58, pp. 167-202.

41. Wolff, P.; Thorstad, R. Force dynamics. In The Oxford Handbook of Causal Reasoning; Waldmann, M.R., Ed.; Oxford University Press: New York, NY, USA, 2017; pp. 147-167.

42. Runeson, S. Perception of biological motion: The KSD-principle and the implications of a distal versus proximal approach. In Perceiving Events and Objects; Jansson, G., Bergström, S.S., Epstein, W., Eds.; Lawrence Erlbaum Associates: Hillsdale, NJ, USA, 1994; pp. 383-405.

43. Göksun, T.; George, N.R.; Hirsh-Pasek, K.; Golinkoff, R.M. Forces and motion: How young children understand causal events. Child Dev. 2013, 84, 1285-1295. [CrossRef] [PubMed]

44. George, N.R.; Göksun, T.; Hirsh-Pasek, K.; Golinkoff, R.M. Any way the wind blows: Children's inferences about force and motion events. J. Exp. Child Psychol. 2019, 177, 119-131. [CrossRef] [PubMed]

45. Gärdenfors, P. Evolutionary and developmental aspects of intersubjectivity. In Consciousness Transitions: Phylogenetic, Ontogenetic and Physiological Aspects; Liljenström, H., Århem, P., Eds.; Elsevier: Amsterdam, The Netherlands, 2007; pp. $281-305$.

46. Premack, D.; Woodruff, G. Does the chimpanzee have a theory of mind? Behav. Brain Sci. 1978, 1, 515-526. [CrossRef]

47. Tomasello, M. The human adaptation for culture. Annu. Rev. Anthropol. 1999, 28, 509-529. [CrossRef]

48. Tomasello, M.; Carpenter, M.; Call, J.; Behne, T.; Moll, H. Understanding and sharing intentions: The origins of cultural cognition. Behav. Brain Sci. 2005, 28, 675-691. [CrossRef]

49. Lombard, M.; Gärdenfors, P. Causal cognition and theory of mind in evolutionary cognitive archaeology. Biol. Theory 2021, 1-19. [CrossRef]

50. Gärdenfors, P.; Lombard, M. Technology led to more abstract causal reasoning. Biol. Philos. 2020, 35, 1-23. [CrossRef]

51. Cléry, J.; Guipponi, O.; Wardak, C.; Hamed, S.B. Neuronal bases of peripersonal and extrapersonal spaces, their plasticity and their dynamics: Knowns and unknowns. Neuropsychologia 2015, 70, 313-332. [CrossRef] [PubMed]

52. Maravita, A.; Iriki, A. Tools for the body (schema). Trends Cogn. Sci. 2004, 8, 79-86. [CrossRef] [PubMed]

53. Calvin, W.H. The unitary hypothesis: A common neural circuitry for novel manipulations, language, plan-ahead, and throwing. In Tools, Language, and Cognition in Human Evolution; Gibson, K.R., Ingold, T., Eds.; Cambridge University Press: Cambridge, UK, 1993; pp. 230-250.

54. Preutz, J.D.; Bertolani, P. Savanna chimpanzees, Pan troglodytes verus, hunt with tools. Curr. Biol. 2007, 17, 412-417. [CrossRef] [PubMed]

55. Roach, N.T.; Venkadesan, M.; Rainbow, M.J.; Lieberman, D.E. Elastic energy storage in the shoulder and the evolution of high-speed throwing in Homo. Nature 2013, 498, 483-487. [CrossRef] [PubMed]

56. Gärdenfors, P.; Lombard, M. Causal cognition, force dynamics and early hunting technologies. Front. Psychol. 2018, 9, 87. [CrossRef]

57. Conard, N.J.; Serangeli, J.; Bigga, G.; Rots, V. A 300,000-year-old throwing stick from Schöningen, northern Germany, documents the evolution of human hunting. Nat. Ecol. Evol. 2020, 4, 690-693. [CrossRef] [PubMed]

58. Hardy, B.L.; Moncel, M.; Daujeard, C.; Fernandes, P.; Béarez, P.; Desclaux, E.; Navarro, M.G.C.; Puaud, S.; Gallotti, R. Impossible Neanderthals? Making string, throwing projectiles and catching small game during Marine Isotope Stage 4 (Abri du Maras, France). Quat. Sci. 2013, 82, 23-40. [CrossRef]

59. Thieme, H. Lower Palaeolithic hunting spears from Germany. Nature 1997, 385, 807-810. [CrossRef]

60. Milks, A.; Parker, D.; Pope, M. External ballistics of Pleistocene hand-thrown spears: Experimental performance data and implications for human evolution. Sci. Rep. 2019, 9, 820. [CrossRef] [PubMed] 
61. Wilkins, J.; Schoville, B.J.; Brown, K.S.; Chazan, M. Evidence for early hafted hunting technology. Science 2012, 338, 942-946. [CrossRef] [PubMed]

62. Bradfield, J.; Wadley, L.; Lombard, M. Southern African arrow poison recipes, their ingredients and implications for Stone Age archaeology. S. Afr. Humanit. 2015, 27, 29-64.

63. Gärdenfors, P. The Geometry of Meaning: Semantics Based on Conceptual Spaces; MIT Press: Cambridge, MA, USA, 2014.

64. Gärdenfors, P.; Jost, J.; Warglien, M. From actions to events: Three constraints on event mappings. Front. Psychol. 2018, 9. [CrossRef]

65. Gärdenfors, P.; Warglien, M. Using conceptual spaces to model actions and events. J. Semant. 2012, 29, 487-519. [CrossRef]

66. Radvansky, G.A.; Zacks, J.M. Event Cognition; Oxford University Press: Oxford, UK, 2014.

67. Warglien, M.; Gärdenfors, P.; Westera, M. Event structure, conceptual spaces and the semantics of verbs. Theor. Linguist. 2012, 38, 159-193. [CrossRef]

68. Dowty, D. Thematic proto-roles and argument selection. Language 1991, 67, 547-619. [CrossRef]

69. Levin, B.; Rappaport Hovav, M. Argument Realization; Cambridge University Press: Cambridge, UK, 2005.

70. Casati, R.; Varzi, A. Event concepts. In Understanding Events: From Perception to Action New; Shipley, T.F., Zacks, J., Eds.; Oxford University Press: York, UK, 2008; pp. 31-54.

71. Zacks, J.M.; Tversky, B. Event structure in perception and conception. Psychol. Bull. 2001, 127, 3-21. [CrossRef]

72. Gärdenfors, P.; Osta Vélez, M. Generics as expectations. 2021, submitted.

73. Granger, C.W. Investigating causal relations by econometric models and cross-spectral methods. Econom. J. Econom. Soc. 1969, 37, 424-438. [CrossRef]

74. Salmon, W.C. Scientific Explanation and the Causal Structure of the World; Princeton University Press: Princeton, NJ, USA, 1984.

75. Talmy, L. Force dynamics in language and cognition. Cogn. Sci. 1988, 12, 49-100. [CrossRef]

76. Liebenberg, L. The Art of Tracking; David Philip Publishers: Cape Town, South Africa, 1990.

77. Lombard, M.; Gärdenfors, P. Tracking the evolution of causal cognition in humans. J. Anthropol. Sci. 2017, 95, 219-234. [PubMed]

78. Gulz, A. The Planning of Action as a Cognitive and Biological Phenomenon; Lund University Cognitive Studies 2: Lund, Sweden, 1991.

79. Osvath, M.; Gärdenfors, P. Oldowan Culture and the Evolution of Anticipatory Cognition; Lund University Cognitive Studies 122: Lund, Sweden, 2005.

80. Premack, D. Human and animal cognition: Continuity and discontinuity. Proc. Natl. Acad. Sci. USA 2007, 104, 13861-13867. [CrossRef]

81. Suddendorf, T.; Corballis, M.C. The evolution of foresight: What is mental time travel and is it unique to humans? Behav. Brain Sci. 2007, 30, 299-351. [CrossRef]

82. Tulving, E. Episodic memory: From mind to brain. Annu. Rev. Psychol. 2002, 53, 1-25. [CrossRef]

83. Mulcahy, N.J.; Call, J. How great apes perform on a modified trap-tube task. Anim. Cogn. 2006, 9, 193-199. [CrossRef] [PubMed]

84. Osvath, M.; Osvath, H. Chimpanzee (Pan troglodytes) and orangutan (Pongo abelii) forethought: Self-control and pre-experience in the face of future tool use. Anim. Cogn. 2008, 11, 661-674. [CrossRef]

85. Osvath, M. Spontaneous planning for future stone throwing by a male chimpanzee. Curr. Biol. 2009, 19, R190-R191. [CrossRef] [PubMed]

86. Osvath, M. Great ape foresight is looking great. Anim. Cogn. 2010, 13, 777-781. [CrossRef] [PubMed]

87. Raby, C.; Alexis, D.; Dickinson, A.; Clayton, N.S. Planning for the future by western scrub-jays. Nature 2007, 445, 919-921. [CrossRef] [PubMed]

88. Correia, S.P.; Dickinson, A.; Clayton, N.S. Western scrub-jays anticipate future needs independently of their current motivational state. Curr. Biol. 2007, 17, 856-861. [CrossRef] [PubMed]

89. Schacter, D.L.; Addis, D.R. The cognitive neuroscience of constructive memory: Remembering the past and imagining the future. Philos. Trans. R. Soc. B Biol. Sci. 2007, 362, 773-786. [CrossRef] [PubMed]

90. Tulving, E. Memory and consciousness. Can. Psychol. 1985, 26, 1. [CrossRef]

91. Martin, V.C.; Schacter, D.L.; Corballis, M.C.; Addis, D.R. A role for the hippocampus in encoding simulations of future events. Proc. Natl. Acad. Sci. USA 2011, 108, 13858-13863. [CrossRef]

92. Bellmund, J.L.; Gärdenfors, P.; Moser, E.I.; Doeller, C.F. Navigating cognition: Spatial codes for human thinking. Science 2018, 362. [CrossRef]

93. Kuhrt, D.; John, N.R.S.; Bellmund, J.L.; Kaplan, R.; Doeller, C.F. An immersive first-person navigation task for abstract knowledge acquisition. Sci. Rep. 2021, 11, 1-16. [CrossRef] [PubMed]

94. Coolidge, F.L.; Haidle, M.N.; Lombard, M.; Wynn, T. Bridging theory and bow hunting: Human cognitive evolution and archaeology. Antiquity 2016, 90, 219-228. [CrossRef]

95. Rafetseder, E.; Schwitalla, M.; Perner, J. Counterfactual reasoning: From childhood to adulthood. J. Exp. Child Psychol. 2013, 114, 389-404. [CrossRef]

96. Markovits, H. On the road toward formal reasoning: Reasoning with factual causal and contrary-to-fact causal premises during early adolescence. J. Exp. Child Psychol. 2014, 128, 37-51. [CrossRef] [PubMed]

97. Baird, A.A.; Fugelsang, J.A. The emergence of consequential thought: Evidence from neuroscience. Philos. Trans. R. Soc. B Biol. Sci. 2004, 359, 1797-1804. 
98. Wolff, P.; Barbey, A.K.; Hausknecht, M. For want of a nail: How absences cause events. J. Exp. Psychol. Gen. 2010, $139,191-221$. [CrossRef]

99. Clark, H.H. Using Language; Cambridge University Press: Cambridge, UK, 1996.

100. Gärdenfors, P.; Brinck, B.; Osvath, M. Coevolution of cooperation, cognition and communication. In New Perspectives of the Symbolic Species; Stjernfelt, F., Deacon, T., Schilhab, T., Eds.; Springer: Berlin/Heidelberg, Germany, 2012; pp. $193-222$.

101. Gärdenfors, P.; Osvath, M. Prospection as a cognitive precursor to symbolic communication. In Evolution of Language: Biolinguistic Approaches; Larson, R.K., Déprez, V., Yamakido, H., Eds.; Cambridge University: Cambridge, UK, 2010; pp. $103-114$.

102. Morrison, D.M. Disambiguated indexical pointing as a tipping point for the explosive emergence of language among human ancestors. Biol. Theory 2020, 15, 196-211. [CrossRef]

103. Tomasello, M.; Carpenter, M.; Liszkowski, U. A new look at infant pointing. Child Dev. 2007, 78, 705-722. [CrossRef] [PubMed]

104. Lyn, H.; Russell, J.L.; Leavens, D.A.; Bard, K.A.; Boysen, S.T.; Schaeffer, J.A.; Hopkins, W.D. Apes communicate about absent and displaced objects: Methodology matters. Anim. Cogn. 2014, 17, 85-94. [CrossRef]

105. Bates, E.; Camaioni, L.; Volterra, V. The acquisition of performatives prior to speech. Merrill Palmer Q. Behav. Dev. 1975, 21, 205-224.

106. Bühler, K. Sprachtheorie: Die Darstellungsfunktion der Sprache; Fischer: Stuttgart, Germany, 1982.

107. Haviland, J.B. Pointing, gesture space and mental maps. In Language and Gesture; McNeill, D., Ed.; Cambridge University Press: Cambridge, UK, 2000; pp. 13-46.

108. McNeill, D. Gesture and Thought; University of Chicago Press: Chicago, IL, USA, 2005.

109. Gärdenfors, P.; Warglien, M. The development of semantic space for pointing and verbal communication. In Conceptual Spaces and the Construal of Spatial Meaning: Empirical Evidence from Human Communication; Hudson, J., Magnusson, U., Paradis, C., Eds.; Cambridge University Press: Cambridge, UK, 2013; pp. $29-42$.

110. Bickerton, D. Language and Species; University of Chicago Press: Chicago, IL, USA, 1990.

111. Dessalles, J.-L. Why We Talk; Oxford University Press: Oxford, UK, 2007.

112. Everett, D. How Language Began: The Story of Humanity's Greatest Invention; Norton: New York, NY, USA, 2017.

113. Gärdenfors, P. The role of cooperation in the evolution of protolanguage and language. In Evolution of Mind, Brain, and Culture; Hatfield, G., Pittman, H., Eds.; University of Pennsylvania Museum of Archaeology and Anthropology: Philadephia, PA, USA, 2013; pp. 193-216.

114. Gärdenfors, P. The cognitive and communicative demands of cooperation. In Games, Actions and Social Software; van Eijck, J., Verbrugge, R., Eds.; Springer: Berlin/Heidelberg, Germany, 2012; Volume 7010, pp. 164-183.

115. Zlatev, J.; Persson, T.; Gärdenfors, P. Bodily mimesis as 'the missing link' in human cognitive evolution. Lund Univ. Cogn. Stud. 2005, 121, 1-45.

116. Brinck, I.; Gärdenfors, P. Cooperation and communication in apes and humans. Mind Lang. 2003, 18, 484-501. [CrossRef]

117. Gärdenfors, P. Cooperation and the evolution of symbolic communication. In The Evolution of Communication Systems; Oller, K., Griebel, U., Eds.; MIT Press: Cambridge, MA, USA, 2004; pp. 237-256.

118. Tylén, K.; Weed, E.; Wallentin, M.; Roepstorff, A.; Frith, C.D. Language as a tool for interacting minds. Mind Lang. 2010, 25, 3-29. [CrossRef]

119. Meldrum, J.; Hilton, C.E. From Biped to Strider: The Emergence of Modern Human Walking, Running, and Resource Transport; Meldrum, J., Hilton, C.E., Eds.; Springer Science \& Business Media: Berlin/Heidelberg, Germany, 2004.

120. Isaac, L.G. The earliest archaeological traces. In Cambridge History of Africa, Volume 1; Clark, J.D., Ed.; Cambridge University Press: Cambridge, UK, 1982; pp. 157-247.

121. Toth, N. The Oldowan reassessed: A close look at early stone artefacts. J. Archeol. Sci. 1985, 12, 101-120. [CrossRef]

122. Donald, M. Key cognitive preconditions for the evolution of language. Psychon. Bull. Rev. 2016, 24. [CrossRef]

123. Gärdenfors, P.; Högberg, A. The archaeology of teaching and the evolution of Homo docens. Curr. Anthropol. 2017, 58, 188-201. [CrossRef]

124. Kline, M.A. How to learn about teaching: An evolutionary framework for the study of teaching behavior in humans and other animals. Behav. Brain Sci. 2015, 38, 1-17. [CrossRef] [PubMed]

125. Boesch, C. Cooperative hunting roles among Tai chimpanzees. Hum. Nat. 2002, 13, 27-46. [CrossRef]

126. Sterelny, K. The Evolved Apprentice; MIT Press: Cambridge, MA, USA, 2012.

127. Számadó, S. Pre-hunt communication provides context for the evolution of early human language. Biol. Theory 2010, 5, 366-382. [CrossRef]

128. Smith, E.A. Communication and collective action: Language and the evolution of human cooperation. Evol. Hum. Behav. 2010, 31, 231-245. [CrossRef]

129. Nowak, M.A.; Sigmund, K. Evolution of indirect reciprocity. Nature 2005, 437, 1291-1298. [CrossRef] [PubMed]

130. Leimar, O.; Hammerstein, P. Evolution of cooperation through indirect reciprocity. Proc. R. Soc. Lond. Ser. B Biol. Sci. 2001, 268, 745-753. [CrossRef] [PubMed]

131. Dunbar, R. Grooming, Gossip and the Evolution of Language; Faber and Faber: London, UK, 1996.

132. Slingerland, I.; Mulder, M.; van der Vaart, E.; Verbrugge, R. A multi-agent systems approach to gossip and the evolution of language. In Proceedings of the 31st Annual Meeting of the Cognitive Science Society (CogSci'09), Amsterdam, The Netherlands, 29 July-1 August 2009; 1 August 2009; pp. 1609-1614. 
133. Semmann, D.; Krambeck, H.J.; Milinski, M. Reputation is valuable within and outside one's own social group. Behav. Ecol. Sociobiol. 2005, 57, 611-616. [CrossRef]

134. Gärdenfors, P. From pantomime to protolanguage. Paradigmi 2020, 38, 251-268.

135. Croft, W. Syntactic Categories and Grammatical Relations: The Cognitive Organization of Information; University of Chicago Press: Chicago, IL, USA, 1991.

136. Goldberg, A.E. Constructions: A Construction Grammar Approach to Argument Sstructure; University of Chicago Press: Chicago, IL, USA, 1995.

137. Langacker, R.W. Foundations of Cognitive Grammar: Theoretical Prerequisites; Stanford University Press: Stanford, CA, USA, 1987; Volume 1.

138. Talmy, L. Toward a Cognitive Semantics, Vol 2: Typology and Process in Concept Structuring; MIT Press: Cambridge, MA, USA, 2001.

139. Croft, W.; Wood, E.J. Construal operations in linguistics and artificial intelligence. Mean. Cogn. Multidiscip. Approach $2000,2,51$.

140. Jackendoff, R. Foundations of Language: Brain, Meaning, Grammar, Evolution; Oxford University Press: Oxford, MS, USA, 2002.

141. Rosen, S.T. The syntactic representation of linguistic events. In The Second Glot International State-of-the-Article Book; De Gruyter Mouton: Berlin, Germany, 2014; pp. 323-366.

142. DeLancey, S. Event construal and case role assignment. In Annual Meeting of the Berkeley Linguistics Society, Proceedings of the Seventeenth Annual Meeting of the Berkeley Linguistics Society: General Session and Parasession on the Grammar of Event Structure; Linguistic Society of America: Washington, DC, USA, 1991; Volume 17, pp. 338-353.

143. Rappaport Hovav, M.; Levin, B. Reflections on manner/result complementarity. In Lexical Semantics, Syntax, and Event Structure; Rappaport Hovav, M., Doron, D., Sichel, I., Eds.; Oxford University Press: Oxford, UK, 2010; pp. 21-38.

144. Rappaport Hovav, M.; Levin, B. Building verb meanings. In The Projection of Arguments: Lexical and Compositional Factors; CSLI Publications: Stanford, CA, USA, 1998; pp. 97-134.

145. Goddard, C.; Wierzbicka, A. Introducing lexical primitives. In Semantic and Lexical Universals: Theory and Empirical Findings; John Benjamins Publishing Company: Amsterdam, The Netherlands, 1994; pp. 31-54.

146. Dixon, R.M.W. Adjective classes in typological perspective. In Adjective Classes: A Cross-Linguistic Typology; Dixon, R.M.V., Aikhenvald, A.Y., Eds.; Oxford University Press: Oxford, UK, 2004; pp. 1-49.

147. Paradis, C. Ontologies and construals in lexical semantics. Axiomathes 2005, 15, 541-573. [CrossRef]

148. Zwarts, J.; Gärdenfors, P. Locative and directional prepositions in conceptual spaces: The role of polar convexity. J. Logic Lang. Inf. 2016, 25, 109-138. [CrossRef] 